\title{
Estimating the Relative Cost Effectiveness of Four Contraceptive Methods in the Prevention of Unwanted Pregnancies within Department of Defense Active-Duty Women
}

\author{
Donald L. Harrison and Karen Ann Sauer
}

\begin{abstract}
OBJECTIVE: To determine the relative cost effectiveness of depo medroxyprogesterone acetate (DMPA), levonorgestrel subdermal implant (LSI), oral tri-phasic tablets (0TPT), and the copper intrauterine device (Cu-IUD) in the prevention of unwanted pregnancies. A further purpose of this research was to estimate the impact each alternative has on the total direct medical costs associated with unwanted pregnancies.
\end{abstract}

DESIGN: Decision analysis, using published clinical outcome probability estimates and actual provider cost data to compare the relative cost effectiveness of the four alternatives.

PATIENTS/SETTING: This research was conducted from the perspective of the provider, Department of Defense (DoD) medical treatment facilities. The patients studied were active-duty women in military service receiving contraceptive services.

MAIN OUTCOME MEASURES: The average cost per patient for four contraceptive measures. All costs are actual costs to the provider and were provided by DoD Resource Management personnel using standard DoD cost accounting reports. Because the time frame considered was less than 12 months, all costs are depicted in same-year dollars.

RESULTS: Costs of providing contraception were much lower for DMPA when compared to OTPT, LSI, and Cu-IUD. Because each alternative is a highly effective therapy, the average cost-effectiveness ratios (cost per successful outcome) were very similar to the average costs.

When the prevention of unwanted pregnancies is considered, DMPA is substantially more cost effective than the other alternatives. Further, both OTPT and Cu-IUD are almost equally cost effective, while LSI is the least cost-effective alternative. Additionally, this research demonstrates that the cost effectiveness of DMPA is not sensitive to variations in costs or probability of failure associated with any of the alternatives assessed. Finally, the economic impact to the provider (in terms of direct medical costs) associated with unwanted pregnancies is highlighted by this research.

KEYwORDS: Contraceptive, DMPA, OTPT, LSI, CU-IUD, Decision analysis

J Managed Care Pharm 1999: 131-36

$\mathrm{P}$ regnancy-related conditions are the primary reason for the hospitalization of active-duty military women. ${ }^{1}$

Although documentation is not available regarding how many pregnancies are planned versus unplanned, a substantial number could be due to contraceptive failure.

It has been reported that approximately $5 \%$ of the U.S. Army's female soldiers became pregnant while serving in Bosnia. ${ }^{2}$ The estimated pregnancy rate for a six-month period during Desert Storm was 2.3\%; the primary reason for evacuating females during Desert Storm was pregnancy. Prior to deployment, limited counseling regarding contraception was provided to these women. Once in the field, oral contraceptive supplies were limited, and women often had to use a different brand or strength from what they had previously used. ${ }^{3}$

Supply problems are not confined to wartime deployments. A recent report by Schwerin and Sack presented the results of interviews with senior Navy health care providers from 36 ships. ${ }^{4}$ On most ships, personnel receive training programs on birth control, sexually transmitted diseases, and the Navy pregnancy policy. However, the providers reported inadequate or inappropriate supplies of such items as contraceptives, pregnancy tests, and sexually transmitted disease test kits. 
Females in the military are relatively young; in fact, nearly half are 25 years of age or younger. ${ }^{5}$ Within the civilian population, this age group relies most frequently on oral contraceptives and condoms as contraceptive methods. ${ }^{6}$ In the military population, the selection of oral contraceptives may be influenced by the lack of availability of other hormonal agents. Only a limited number of military clinics carry the longer-acting agents Depo-Provera and Norplant. ${ }^{3}$ The Tri-Service Formulary lists only six different combined oral contraceptives

Table 1. Summary of Probabilities for the Four Contraceptive Methods

\begin{tabular}{|c|c|c|c|c|}
\hline \multirow[t]{2}{*}{ Outcome } & \multicolumn{4}{|c|}{ Probability } \\
\hline & OTPT & DMPA & Cu-IUD & LSI \\
\hline $\begin{array}{l}\text { Success } \\
\text { (pregnancy avoided) }\end{array}$ & 0.94 & 0.996 & 0.96 & 0.9995 \\
\hline $\begin{array}{l}\text { Failure } \\
\text { (pregnancy not avoided) }\end{array}$ & 0.06 & 0.004 & 0.04 & 0.0005 \\
\hline Gall bladder disease & 0.00093 & $\mathrm{NA}$ & $\mathrm{NA}$ & $\mathrm{NA}$ \\
\hline Pulmonary embolism & 0.00011 & NA & NA & NA \\
\hline Stroke & 0.00003 & $\mathrm{NA}$ & NA & NA \\
\hline MI (nonsmoker) & 0.000006 & $\mathrm{NA}$ & $\mathrm{NA}$ & $\mathrm{NA}$ \\
\hline Mi (light smoker) & 0.00001 & NA & $\mathrm{NA}$ & $\mathrm{NA}$ \\
\hline MI (heavy smoker) & 0.00001 & $\mathrm{NA}$ & $\mathrm{NA}$ & NA \\
\hline Irregular bleeding & NA & 0.025 & $\mathrm{NA}$ & 0.025 \\
\hline PID & NA & $\mathrm{NA}$ & 0.083 & NA \\
\hline Perforation & NA & NA & 0.0004 & $\mathrm{NA}$ \\
\hline Expulsion & $\mathrm{NA}$ & NA & 0.06 & $\mathrm{NA}$ \\
\hline $\begin{array}{l}\text { Infection at } \\
\text { insertion sire }\end{array}$ & NA & NA & NA & 0.0008 \\
\hline No adverse events & 0.998904 & 0.975 & 0.8566 & 0.9742 \\
\hline Ectopic pregnancy & 0.01 & 0.13 & 0.04 & 0.14 \\
\hline Spontaneous abortion & 0.127 & 0.127 & 0.25 & 0.127 \\
\hline Induced abortion & 0.41 & 0.41 & 0.41 & 0.41 \\
\hline Term delivery & 0.453 & 0.333 & 0.30 & 0.323 \\
\hline $\begin{array}{l}\text { Normal (vaginal) } \\
\text { delivery }\end{array}$ & 0.772 & 0.772 & 0.772 & 0.772 \\
\hline Caesarean delivery & 0.228 & 0.228 & 0.228 & 0.228 \\
\hline Normal infant care & 0.947 & 0.947 & 0.947 & 0.974 \\
\hline Intensive infant care & 0.053 & 0.053 & 0.053 & 0.053 \\
\hline
\end{tabular}

NA=Not applicable

Source: Hatcher et al., 1994; Harlap et al., 1991 as options for contraception. ${ }^{7}$ Furthermore, compliance is a problem with oral contraceptives even under the best of conditions. Poor compliance leads to failure rates of approximately $6 \%$ for all ages, under typical use; this number is higher for women under 25 years of age. ${ }^{8}$ It is highly probable that in deployed situations, such as Bosnia and the Persian Gulf, the failure rate is even higher.

When a military woman is found to be pregnant during a tour of duty or shipboard assignment, she is airlifted to a site with additional medical support, because field and ship physicians tend to have limited gynecological training. ${ }^{4.9}$ Additionally, it is necessary to remove the woman from environmental elements such as chemicals, heat, and noise that may pose a hazard to the woman and the fetus. ${ }^{10}$

At a recent Uniformed Services University of Health Sciences (USUHS) conference it was announced that money spent to improve pregnancy prevention efforts would reduce the large expenditures needed to care for unintended pregnancy. ${ }^{10}$ However, the amount needed is not known.

The purpose of this research was to estimate the relative cost effectiveness of depo medroxyprogesterone acetate (DMPA), levonorgestrel subdermal implant (LSI), oral tri-phasic tablets (OTPT), and the copper intrauterine device (CuIUD) in the prevention of unwanted pregnancies. These methods were chosen because of their relatively low failure rate and their reversibility. A further purpose of this research was to estimate the impact each alternative has on the total direct medical costs associated with unwanted pregnancies. Finally, this research was conducted from the perspective of the provider (Department of Defense medical treatment facilities).

\section{METHODS}

\section{Model Development}

This research considered four alternatives in the prevention of unwanted pregnancies: OTPT, injectable DMPA, LSI, and the Cu-IUD. The relative cost effectiveness of the alternatives was modeled using decision analysis techniques described by Weinstein et al. ${ }^{11}$ Decision trees for the four alternatives were constructed and the cost effectiveness of all alternatives was modeled using the decision analysis software DATA (version 3.0) for Windows. ${ }^{12}$ The decision trees used success (unwanted pregnancy avoided) or failure (unwanted pregnancy not avoided) as the clinical outcomes. The model also incorporated the significant adverse events associated with the therapies considered. ${ }^{8,13}$ The model used in this research covered the period up to and including infant discharge after delivery resulting from contraceptive failure. Several significant adverse events were incorporated in the model for OTPT: gall bladder disease, pulmonary embolism, stroke, and myocardial infarction (MI). It should be noted that the risk of MI is not uniform across the population of OTPT users. Therefore, published data were used that considered the incremental risk incurred by smoking 
status (heavy, light, and nonsmoker) weighted by the relative proportions of smoking usage in the population. Irregular bleeding was the only adverse event associated with DMPA that was incorporated into the model. For IUDs, pelvic inflammatory disease (PID), uterine perforation during insertion, and expulsion (requiring reinsertion) were used. The adverse events included for LSI were irregular bleeding and infection at the site of implant. The treatment path for the failure of a contraceptive method was very similar for all alternatives considered. However, it is important to note that the probabilities for some treatment paths (nodes) were not the same for each alternative. For each failure, four options (nodes) were incorporated into the decision tree: ectopic pregnancy, spontaneous abortion, induced abortion, or term delivery. For those unwanted pregnancies brought to term, normal (vaginal) delivery or Caesarean delivery were the two options considered. Finally, normal infant care or intensive infant care provided to infants after delivery and prior to hospital discharge were incorporated.

\section{Decision Analysis}

Decision analysis was used to calculate the average cost per patient (expected value) and probability of successful outcome (pregnancy avoided). The cost effectiveness of all alternatives was modeled using the decision analysis software DATA (version 3.0) for Windows. ${ }^{12}$ Probability estimates employed in the various nodes of the decision tree(s) were derived from published literature. ${ }^{8,13}$ Average cost-effectiveness ratios were calculated by dividing the average cost by the probability of successful outcome for each alternative. Incremental cost-effectiveness ratios were calculated for each alternative compared to DMPA. The incremental cost-effectiveness ratio is very important in the decision-making process because it reflects the additional cost incurred for each additional successful outcome achieved compared to DMPA. ${ }^{34}$

\section{Probability Estimates}

Conditional probabilities for all outcomes based on published data were inserted into the decision analysis model. ${ }^{8.13}$ The probability estimates were rounded where necessary so that the probabilities at each node summed to one. Table 1 provides a summary of probability estimates for each alternative used in the decision analysis. Note that the probability estimates for success and failure used in these analyses are reported "typical use" or effectiveness data and are not "ideal use" or efficacy data. The estimates used in this analysis have been adjusted by the general rate of pregnancy with active coitus. ${ }^{8.13}$ The estimates of failure and success for all alternatives were reviewed by OB/GYN consultants and found to be appropriate estimates of normal effectiveness. Because of the short time frame, discontinuation rates were not analyzed.

\section{Cost Estimates}

Because this research was undertaken from the provider's perspective, only direct medical costs were included. All costs are actual; they were provided by Department of Defense
(DoD) Resource Management personnel using standard DoD cost accounting reports. Further, because the time frame considered in this research was less than 12 months, all costs are in same-year dollars. Table 2 provides a detailed account of all cost components and their dollar values. The costs for OTPT and injectable DMPA are total annual costs, including product acquisition, physician visit, and administration costs, but not laboratory costs. The total annual cost for OTPT was $\$ 119$, which included $\$ 4.50$ per cycle (13 cycles per year) and a $\$ 60$ physician visit. The total annual cost for DMPA was $\$ 207$, which included $\$ 18$ per injection (four injections), a $\$ 60$ initial physician visit, and three $\$ 25$ medication administration costs. Costs for the Cu-IUD and LSI include product and insertion costs but not removal costs. The law prohibits the DoD from performing or paying for induced abortions and therefore there is no cost to the DoD for this outcome.

\section{Sensitivity Analyses}

A series of one-way sensitivity analyses was conducted for each cost-effectiveness ratio modeled. The analyses were chosen because they target key components of the decisionmaking process. ${ }^{15,16}$ Three sensitivity analyses compared the

\section{Table 2. Summary Cost Data}

\begin{tabular}{l|c}
\hline Component & Cost \\
\hline DMPA & $\$ 207$ \\
\hline LSI & $\$ 500$ \\
\hline OTPT & $\$ 119$ \\
\hline Cu-IUD & $\$ 200$ \\
\hline Gall bladder disease & $\$ 5,500$ \\
\hline Pulmonary embolism & $\$ 7,500$ \\
\hline Stroke & $\$ 17,500$ \\
\hline MI & $\$ 14,750$ \\
\hline Irregular bleeding & $\$ 70$ \\
\hline PID & $\$ 100$ \\
\hline Infection at site of implant & $\$ 90$ \\
\hline Expulsion & $\$ 200$ \\
\hline Ectopic pregnancy & $\$ 5,000$ \\
\hline Spontaneous abortion & $\$ 1,100$ \\
\hline Induced abortion & $\$ 0$ \\
\hline Normal (vaginal) delivery & $\$ 4,000$ \\
\hline Caeserean delivery & $\$ 7,500$ \\
\hline Normal infant care & $\$ 1,000$ \\
\hline Intensive infant care & $\$ 25,000$ \\
\hline Source: DoD cost accounting reports, 1997. & \\
\hline
\end{tabular}


Table 3. Average and Incremental Cost-Effectiveness Ratios

\begin{tabular}{l|c|c|c|c|c}
\hline Comparison & Average cost per patient & $\begin{array}{c}\text { Total cost per cohort } \\
\text { (millions) }\end{array}$ & $\begin{array}{c}\text { Total number of } \\
\text { pregnancies avoided }\end{array}$ & $\begin{array}{c}\text { Average cost effectiveness } \\
\text { effectiveness }\end{array}$ \\
\hline DMPA & $\$ 221$ & $\$ 2.21$ & 9,960 & $\$ 222$ \\
\hline OTPT & $\$ 329$ & $\$ 3.29$ & 9,400 & $\$ 350$ \\
\hline CU-IUD & $\$ 325$ & $\$ 3.25$ & 9,600 & $\$ 1,929$ \\
\hline LSI & $\$ 503$ & $\$ 5.03$ & 9,995 & $\$ 2,889$ & $\$ 503$ \\
\hline
\end{tabular}

Average cost effectiveness = Average cost per successful outcome (pregnancy avoided)

Incremental cost effectiveness = Cost incurred to achieve an additional successful outcome using DMPA when compared to an alternate method.

cost effectiveness of DMPA to OTPT. The first determined sensitivity to variations in the cost of DMPA, from $\$ 100$ to $\$ 400$; the second, the sensitivity of the decision to variations in probability of failure associated with OTPT, which varied from 0.01 to 0.15 ; and the third analysis, the sensitivity to variations in the cost of OTPT, from $\$ 50$ to $\$ 200$.

To compare the cost effectiveness of DMPA and LSI, two sensitivity analyses were completed. The first determined the sensitivity of variations in probability of failure associated with DMPA, which varied from 0.001 to 0.15 . The second determined sensitivity to variations in the cost of LSI from $\$ 100$ to $\$ 700$.

To compare the cost effectiveness of DMPA and Cu-IUD, three sensitivity analyses were completed. The first determined sensitivity of the decision to variations in the cost of DMPA, from $\$ 100$ to $\$ 400$; the second, the sensitivity to variations in probability of failure associated with IUDs, from 0.0005 to 0.07 ; and the third, the sensitivity of the decision to variations in probability of failure associated with DMPA, which varied from 0.001 to 0.10 .

The assumptions used in the analyses were that:

$\Delta$ The range of values assigned to each source of medical costs is both appropriate and representative of normal values.

$\Delta$ The alternatives studied are independent of each other.

$\Delta$ All pregnancies resulting from the failure of contraceptive methods are unwanted.

\section{RESULTS}

\section{Cost-Effectiveness Decision Analysis}

The results of the decision analysis are presented in Table 3. The relative cost effectiveness of the four contraceptive alternatives was modeled using pregnancy avoided as the outcome measure (consequence). The total average cost per patient treated with DMPA was $\$ 221$. The average cost per patient using OTPT was $\$ 329$, while the average cost of those patients given LSI and Cu-IUD was $\$ 503$ and $\$ 325$, respectively. Because each alternative is highly effective, the average cost-effectiveness ratios (cost per successful outcome) were very similar to the average costs: $\$ 222$ for
DMPA, $\$ 350$ for OTPT, $\$ 503$ for LSI, and $\$ 338$ for Cu-IUD.

Three incremental cost-effectiveness ratios were calculated. The incremental ratios allow the cost-effectiveness ratio of DMPA to be rationally compared to those of the other three alternatives. The three incremental ratios estimate the average cost incurred to achieve an additional successful outcome (pregnancy avoided) with DMPA when compared to the three remaining alternatives. The incremental cost-effectiveness ratio comparing DMPA to OTPT was $-\$ 1,929$. This negative value indicates that the provider saves on average almost $\$ 1,930$ with each additional successful outcome attained with DMPA compared to OTPT. The incremental ratio comparing DMPA to Cu-IUD was $-\$ 2,889$, indicating that on average the provider saves almost $\$ 2,890$ with each additional successful outcome achieved with DMPA compared to the IUD. However, the incremental ratio comparing DMPA to LSI was $\$ 783$, indicating that on average each additional successful outcome achieved with DMPA, when compared to LSI, will cost the provider almost $\$ 785$.

\section{Sensitivity Analyses}

Eight one-way sensitivity analyses were conducted. When the cost of OTPT is held constant and the cost of DMPA increases above $\$ 330.60$, OTPT becomes the dominant strategy, but when the probability of DMPA failure is held constant and the probability of OTPT failure decreases below 0.027 , DMPA becomes the dominant strategy. Finally, when the cost of DMPA is held constant, it remains the dominant strategy, even when the cost of OTPT is as low as $\$ 50$.

When the probability of LSI failure is held constant and the probability of DMPA failure increases above 0.0871 , the dominant strategy becomes LSI, as it does when the cost of DMPA is held constant and the cost of LSI decreases below $\$ 217.50$.

When the cost of the Cu-IUD is held constant and the cost of DMPA increases above $\$ 318.40$, the Cu-IUD becomes the dominant strategy, as it does when the probability of DMPA failure is held constant and the probability of Cu-lUD failure decreases below 0.00059 , and also when the probability of Cu-IUD failure is held constant and the probability of DMPA failure increases above 0.0368 . 


\section{DISCUSSION}

When active-duty female soldiers are deployed, the choice of contraceptive method used depends on many factors, of which cost effectiveness is only one. A reasonable choice also must consider the stage of the soldier's life and the demands of military duty. In this analysis we compared four contraceptive alternatives for the prevention of unwanted pregnancies in Department of Defense women. The results demonstrate that therapy with DMPA is substantially more cost effective than the other three alternatives considered. As depicted in Table 3, DMPA is more than twice as cost effective than LSI ( $\$ 222$ versus $\$ 505)$. Furthermore, it is substantially more cost effective than either the Cu-IUD (\$338) or OTPT (\$350). While DMPA is clearly more cost effective than the other alternatives, the cost effectiveness of both OTPT and the CU-IUD are almost identical. The higher cost of LSI greatly contributes to its ranking as the least cost-effective alternative.

The results highlight some interesting aspects of costeffectiveness estimation. DMPA is substantially more expensive than OTPT; however, it is also considerably more effective and is associated with less serious (and costly) adverse events. Thus, the difference in costs is more than offset by the difference in effectiveness and safety that make DMPA substantially more cost effective. It is considered the dominant strategy. When DMPA is compared to LSI, DMPA is substantially less expensive, but it is also much less effective. Yet the difference in costs is not offset by the difference in effectiveness.

Therefore, DMPA remains substantially more cost effective and the dominant strategy.

When the average direct medical cost of unwanted pregnancies among the four alternatives was compared, the results were almost identical. The average cost per patient was considerably lower with DMPA (\$221). To highlight the differences in average direct medical costs, costs were calculated using a hypothetical cohort of 10,000 women treated with each alternative. When the cohort is treated with DMPA, the total direct medical cost would average $\$ 2.21$ million with 40 unwanted pregnancies $(9,960$ pregnancies avoided). If the cohort were treated with OTPT, total direct medical cost would average $\$ 3.29$ million with 600 unwanted pregnancies $(9,400$ pregnancies avoided). Treating the cohort with LSI would result in a total direct medical cost averaging $\$ 5.03$ million with only five unwanted pregnancies ( 9,995 pregnancies avoided). Using the $\mathrm{Cu}$-IUD leads to a total direct medical cost averaging $\$ 3.25$ million, with 400 unwanted pregnancies $(9,600$ pregnancies avoided).

An analysis of the incremental cost-effectiveness ratios provides some interesting results. When the cost-effectiveness ratio of DMPA is compared to the ratios for OTPT and the $\mathrm{Cu}$ IUD, the results demonstrate that the achievement of additional successful outcomes (unwanted pregnancies avoided) with DMPA is not accompanied by additional costs; rather, the provider realizes a significant cost savings. However, when the cost-effectiveness ratio of DMPA is compared to that of LSI, the results demonstrate that a cost of almost $\$ 785$ is incurred for each additional successful outcome attained with DMPA. This is expected, because of LSI's significantly higher effectiveness. The incremental cost incurred is acceptable given the substantially higher cost effectiveness and substantially lower total direct medical costs incurred with DMPA. Further, the cost of $\$ 783$ incurred for each additional outcome achieved with DMPA is considerably less than the average cost of a pregnancy (at least $\$ 7,280$ ).

The results of the sensitivity analyses demonstrate the robustness of the decision analysis results. When DMPA and OTPT are compared, the cost of DMPA can increase up to $\$ 330$ before OTPT becomes the dominant strategy. Furthermore, the decision is not sensitive to variation in the probability of OTPT failure, which must decline to less than one-half of the baseline rate (from 0.06 to 0.027 ) before the decision changes. Finally, the decision is not sensitive to variations in the cost of OTPT.

The decision analysis comparing DMPA to LSI was not sensitive. Here the cost of LSI must decrease to less than half of the baseline value (from $\$ 500$ to $\$ 217$ ) before LSI becomes the dominant strategy. Furthermore, the decision is not sensitive to variation in the probability of DMPA failure, which must increase 21 -fold (from 0.004 to 0.0871 ) before the decision changes.

When DMPA and the Cu-IUD are compared, the cost of DMPA can increase significantly, from $\$ 207$ to $\$ 318$, before the Cu-IUD becomes the dominant strategy. The decision is not sensitive to variation in the probability of $\mathrm{Cu}$-IUD failure, which must drop more than 65-fold (from 0.04 to 0.00059 ) before the decision changes. Nor is it sensitive to variations in the probability of DMPA failure, which must increase more than nine-fold (from 0.004 to 0.0368 ) before the decision changes.

The results of this research represent a significant advance in the comparison of four alternatives in the prevention of unwanted pregnancies. The developed model incorporates both the economic and clinical consequences of the alternatives considered, providing the first true cost-effectiveness analysis of DMPA, LSI, OTPT, and the Cu-IUD in the prevention of unwanted pregnancies.

There are some limitations to the research. The time frame used was relatively short (less than one year). This could limit the cost effectiveness of such long-term contraceptive methods as LSI and the Cu-IUD. Additionally, because of the short time period, continuation rates were not included. Nor did this study include costs associated with the removal of the Cu-IUD or an LSI implant. It was felt that placing all the removal costs in the time frame under consideration would unfairly penalize these methods. Overall, the impact of this limitation is minimized by the results of the many sensitivity analyses performed and developing evidence that the discontinuation rate associated with these long-term methods (especially LSI) is 
increasing due to general dissatisfaction and unwanted side effects. ${ }^{8,13}$

An additional limitation arises because only direct medical costs were incorporated into the model, which does not account for possible significant indirect costs associated with unwanted pregnancies. This may be especially true in deployed situations (e.g., Bosnia, Persian Gulf, and Navy sea deployment). The need to maintain a potentially larger-thannormal personnel replacement system and aeromedical evacuation costs associated with an unwanted pregnancy are just two potential system costs not accounted for. Most important, because of the inevitable lag in personnel replacement, the force commander may have to operate with fewer personnel available for duty.

Another possible limitation arises because the research used normal effectiveness (typical use) estimates for OTPT. When female soldiers are deployed, it is reasonable to assume that the probability of failure will increase as a result of many factors: problems resulting from switching products upon deployment; supply problems in general; and most important, decreased compliance due to the stresses of being deployed. This limitation serves to strengthen the cost effectiveness of the other three alternatives.

A final limitation arises because the model used to assess the four alternatives was conceptual and therefore involves the use of decision analysis to model cost effectiveness. While the probabilities used in this study were derived from published research, the economic data were estimations obtained from Resource Management personnel. Actual economic data based on head-to-head clinical comparisons are not available. Therefore, the results of this research are simply predictions. However, the impact of this limitation is mitigated by the results of the sensitivity analyses, which demonstrated that the decision is not sensitive to variations in the cost of any of the four alternatives assessed.

Although the setting for this research was DoD medical treatment facilities, we believe the results can be generalized to most managed care settings. Our effectiveness estimates were derived from the general population. Further, although the cost estimates were specific to the DoD, the results of the sensitivity analyses demonstrate that decision analyses are not sensitive to variations in cost.

\section{CONCLUSIONS}

When active-duty female soldiers are deployed, the choice of contraceptive method used depends on many factors; cost effectiveness is only one. A reasonable choice also must consider the stage of the soldier's life and the demands of military duty. The results of these analyses provide the first true cost-effectiveness analysis of depo medroxyprogesterone acetate (DMPA), levonorgestrel subdermal implant (LSI), oral tri-phasic tablets (OTPT), and the copper intrauterine device (Cu-IUD) in the prevention of unwanted pregnancies. When pregnancy prevention is the desired outcome, DMPA is substantially more cost effective than the other alternatives. Next, both OTPT and the Cu-IUD are almost equally cost effective, while LSI is the least cost-effective alternative. These results also demonstrate that when DMPA is compared to OTPT and the Cu-IUD, each additional successful outcome attained with DMPA is accompanied by significant cost savings. When DMPA is compared to LSI, each additional successful outcome is accompanied by moderate costs to the provider, but these are relatively minor compared to the average cost of an unwanted pregnancy. This research also demonstrates that the cost effectiveness of DMPA is not sensitive to variations in costs or the probability of failure associated with any of the alternatives assessed. Finally, the economic impact of unwanted pregnancies to the provider, in terms of direct medical costs, is highlighted.

\section{$\Delta$}

\section{References}

1. Hoiberg A, White JF. Health status of women in the armed forces [abstract] NHRC Publication, 89-37. San Diego: Naval Health Research Center, 1992

2. Graham B. Pregnancy rate of unit in Bosnia hits army average. The Washington Post 1996 July 23: A:15.

3. Gehlbach D. Contraceptive needs, complications, and new directions for research. Women's Health Issues 1996; 6: 355-58.

4. Schwerin MJ, Sack DM. Shipboard women's health care: provider perceptions [abstract] NHRC Publication, 96-29. San Diego: Naval Health Research Center, 1996

5. Table 2-17A. Age distribution of female military personnel strength (in thousands) and percent figures fiscal years 1992 through present. Directorate for Information Operations and Reports, Office of the Secretary of Defense. Available from: http://webl.whs.osd.mil/mmid/m01/fy96/sms217 ar.htm.

6. Abma JC, Chandra A, Mosher WD, et al. Fertility, family planning, and women's health: new data from the 1995 national survey of family growth. National Center for Health Statistics. Vital Health Stat 23(19), 1997.

7. Tri-service formulary quick reference guide. PEC Update 1997 May 16; 3 : 97-108.

8. Harlap S, Kost K, Forrest JD. Preventing pregnancy, protecting health: a new look at birth control choices in the United States. New York: The Alan Guttmacher Institute, 1991.

9. Military physicians review health challenges of female soldiers. ACOG Newsletter 1996(Sept); 40(9): 1, 10.

10. Moore GR. Reproductive hazards: military policy implications. Paper presented at conference: Women's Health Issues 1996; 6: 363-66.

11. Weinstein MC, Fineberg HV, Elstein AS, et al. Clinical decision analysis. Philadelphia: W.B. Saunders Company, 1980.

12. TreeAge. DATA for Windows. Boston: TreeAge Software, Inc., 1996.

13. Hatcher RA, Trussell J, Stewart F, et al. Contraceptive technology, 15th ed. New York: Irving Publishers, 1994.

14. Bloom BS, Hillman AL, LaMont B, et al. Omeprazole or ranitidine plus metoclopramide for patients with severe erosive oesophagitis: a cost-effectiveness analysis. PharmacoEconomics 1995; 8: 343-49.

15. Briggs A, Sculpher M, Buxton M. Uncertainty in the economic evaluation of health care technologies: the role of sensitivity analysis. Health Economics 1994; 3: 95-104.

16. Petitti DB. Meta-analysis, decision analysis, and cost-effectiveness analysis. New York: Oxford University Press, Inc., 1994: 187-96. 